\% Science and Engineering Research Council

Q Rutherford Appleton Laboratory

Chilton DIDCOT Oxon OX11 0QX

RAL-92-053

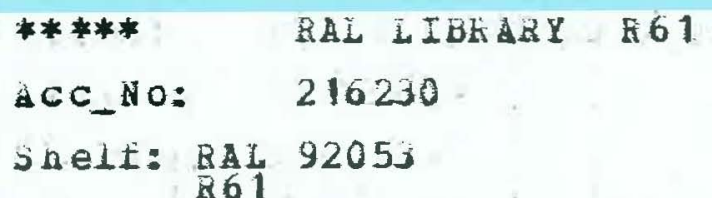

Collective Interactions Between Neutrinos and Dense Plasmas

R J Bingham J M Dawson and J J Su

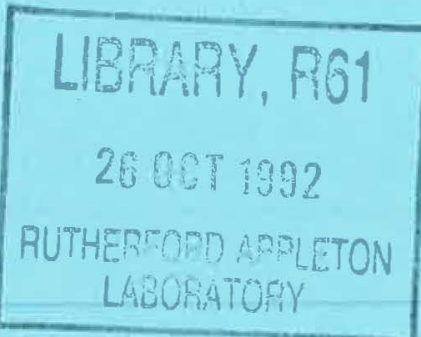

August 1992 
Science and Engineering Research Council

"The Science and Engineering Research Council does not accept any responsibility for loss or damage arising from the use of information contained in any of its reports or

in any communication about its tests or investigations" 


\title{
Collective Interactions Between Neutrinos and Dense Plasmas
}

\author{
R.J. Bingham; J.M. Dawson; and J.J. Su ${ }^{\ddagger}$
}

\begin{abstract}
A new interaction mechanism is described between neutrinos and dense plasmas. With the unification of the electromagnetic and weak forces, analogous processes should occur for intense neutrino fluxes as for photon fluxes. Intense EM waves excite parametric instabilities in nonlinear media and plasma in particular. Therefore, sufficiently intense neutrino fluxes should also cause similar parametric instabilities in dense plasmas. An important example is the production of Langmuir plasmons and lower energy neutrinos. In plasma physics, for electromagnetic waves, the process is known as stimulated Raman scattering and it greatly increases the interaction of the light with the plasma. We propose that the analogous process, for neutrinos, occurs in the plasma surrounding the core of a supernova due to the immense neutrino flux there and the fact that at some distance from the core the flux is strongly unidirectional. We develop a theory for stimulated scattering of neutrinos in plasmas based on the index of refraction for neutrinos which depends on electron density, conservation of energy and momentum for neutrinos plus plasmons and wave damping; we estimate approximate growth rates. We find that the process should, indeed, go between 10 and 100 core radii. We propose that the process plays an important role in supernova dynamics and particularly in the production of the outward going shock.
\end{abstract}

\section{Introduction}

The interaction of neutrinos with matter is an important topic in high energy astrophysics. The question about how supernova explode is still an unsolved problem and depends critically on the transport of neutrinos within the star. During the implosion phase or collapse electron capture occurs, i.e., a proton and electron coalesce to yield a neutron and a neutrino. The super-dense core is optically thick (larger than an absorption length) to the neutrinos and a thermal distribution results with a temperature of about $20 \mathrm{Mev}$. Due to the density fall-off as one leaves the core, there is a surface (known as the neutrino sphere ${ }^{1}$ ), which radiates neutrinos more or less like a black body at this temperature $\left(\sim 10^{34}\right.$ watts per $\mathrm{cm}^{2}$ ). The neutrinos carry away energy and entropy allowing the collapse process to accelerate. It is widely recognized ${ }^{1}$ that more than $1 \%$ of the total neutrino energy flux must be absorbed by electrons in the layers surrounding the super-dense core of the star to produce sufficient electron pressure to eject the outer part of the star leaving only enough mass

\footnotetext{
"Rutherford Appleton Laboratory, Chilton, Didcot, Oxon, OX11 0QX

${ }^{\dagger}$ University of California, Los Angeles, Physics Department Los Angeles, CA 90024-1547

${ }^{\ddagger}$ Institute of Space Science, National Central University, Chung-Li, Taiwan
} 
to form a neutron star. Otherwise no super-novae would occur and only black holes would result. Previous studies ${ }^{2}$ have concentrated on collisional energy losses between neutrinos and fermions, the loss rate, however, is marginal to produce the required electron heating. In this paper we propose an entirely new neutrino interaction mechanism based on the coupling between the neutrinos and collective plasma oscillations in the dense plasma surrounding the core, namely stimulated neutrino-plasmon scattering. The interaction between electrons and neutrinos takes place through the electroweak interaction ${ }^{3}$, the electrons interacting via both electromagnetism and weak interactions and the neutrinos interacting only through the weak interaction. The stimulated scattering process described in this paper has an analogue with laser plasma interactions ${ }^{4}$, in this case the intense photon field of the laser couples to plasma oscillations producing effects such as stimulated Raman and Brillouin scatterings ${ }^{4}$ and the parametric decay process ${ }^{4}$ where a photon is annihilated. Processes such as selffocusing and filamentation instabilities belong to the class of modulational instabilities, are also possible, and are described in terms of a nonlinear refractive index or susceptibility. In fact all material substances interact nonlinearly with intense radiation and the interaction with an intense neutrino flux should follow similar ideas.

The many body interaction between neutrinos and electrons in a plasma is no exception and results in polarization effects allowing the possibility of collective interactions between electron plasma oscillations and the neutrino fields. Stimulated scattering of neutrinos in a dense plasma into either a lower energy neutrino of the same type or a different neutrino and a photon or plasma oscillation is a strong possibility provided the neutrino flux is intense enough to overcome damping of the wave modes. In other words the growth rate of this nonlinear instability must be greater than a threshold value determined by the damping of the resultant modes. In such stimulated scattering processes the neutrino energy flux will decrease while conserving total lepton number, the interaction being determined by the following energy and momentum relationships,

$$
\omega_{\nu o}=\omega_{\nu 1}+\omega_{p} \quad \underline{k}_{\nu o}=\underline{k}_{\nu 1}+\underline{k}_{p}
$$

where $\omega$ and $k$ are the frequencies and wavenumbers of the wave fields (for the neutrino and Langmuir plasmons) subscripts $\nu o, \nu 1$, and $p$ refer to the incident neutrino scattered neutrino and the plasma wave respectively. The interaction we consider is described by a three point interaction as opposed to the more common four-point interaction.

The nonlinear coupling between the neutrino field and the Langmuir plasmon field is easily obtainable from the dispersion relation given by Equation (5) of Bethe ${ }^{5}$ which is

$$
E^{2}-p^{2} c^{2}-m^{2} c^{4}-2 E V=0
$$

where $E$ is normalized energy, $p$ is normalized momentum, $m$ is the normalized neutrino rest mass, and $V$ is equivalent to a potential energy $V=G \sqrt{2} n_{e}$ (where $n_{e}$ is the electron number density) and $G$ is the Fermi constant of the weak interaction. The effects of linear dispersion and amplitude nonlinearity can be studied within the framework of a nonlinear Klein-Gordon equation obtained by making the following substitutions

$$
p \rightarrow-i \hbar \frac{\partial}{\partial x}, E \rightarrow i \hbar \frac{\partial}{\partial t}
$$


in Eq. (4) forming the following wave equation for the neutrino field $\delta \Psi$

$$
\left(\hbar^{2} c^{2} \frac{\partial^{2}}{\partial x^{2}}-m^{2} c^{4}-\hbar^{2} \frac{\partial^{2}}{\partial t^{2}}\right) \delta \Psi=2 i V \hbar \frac{\partial \delta \Psi}{\partial t}
$$

where $V$ is now determined by $n_{e}$ (electron number density per unit volume) and is related to the refractive index of the medium. $\delta \Psi$ is the neutrino amplitude wave function normalized so that the neutrino energy density is $|\delta \Psi|^{2} / 4 \pi,\left(|\delta \Psi|^{2}\right)$ is proportional to the neutrino density times their energy $(\hbar \omega)$. Equation (3) is the Klein-Gordon equation describing the neutrino field coupling into the plasma medium through the potential $\mathrm{V}$, it is similar to the wave equation for electromagnetic waves coupling to a plasma medium. To go from the electromagnetic case to the weak interaction case, one replaces ${ }^{6} \frac{4 \pi e^{2}}{q^{2}}$ by $\frac{4 G}{\sqrt{2}}$.

Writing $\delta \Psi=\delta \Psi_{o}+\delta \Psi_{1}$, where subscript $o, 1$ refers to the incident and scattered field, and using the conservation relations, Equation (1).

Equation (3) can be written in the form

$$
\left(\hbar^{2} c^{2} \frac{\partial^{2}}{\partial x^{2}}-\hbar^{2} \frac{\partial^{2}}{\partial t^{2}}-\tilde{m}^{2}\right) \delta \Psi_{1}=i 2 \sqrt{2} G \delta n_{e} \hbar \frac{\partial \delta \Psi_{o}}{\partial t}
$$

where $\delta n_{e}$ is the perturbed electron density $\left(n_{e}=n_{o}+\delta n_{e}\right)$ varying as $\sim e^{i\left(k_{p} x-\omega_{p} t\right)}$. Equation (5) describes the nonlinear interaction between the incident neutrino field, $\delta \Psi_{o}$, and the plasma wave $\delta n_{e}$ producing a scattered signal at $\delta \Psi_{1}$ and $\tilde{m}^{2}=m^{2} c^{4}+2 V_{o} \hbar \omega_{1}$, with $V_{o}=\sqrt{2} G n_{o}$ being the potential associated with the mean plasma density, $n_{o}$.

From Equation (4) and assuming the amplitudes can be written as a slowly varying part, due to the nonlinear interaction, times a high frequency phase, the time derivative of the slowly varying part of the amplitude, $\delta \Psi_{1}$ is given by

$$
\hbar \frac{\partial \delta \Psi_{1}}{\partial t}=-i \sqrt{2} G \delta n_{e} \delta \Psi_{o}
$$

where we have assume $\omega_{\nu 0} \simeq \omega_{\nu 1}$.

We can also obtain an analogous equation for $\frac{\partial \delta n_{e}}{\partial t}$ in terms of $\delta \Psi_{1, o}$ by using Equation (5) and conservation of momentum. From conservation of momentum (in the case of forward scattering, i.e., $k_{p}=k_{\nu o}-k_{\nu 1}$ ), using the fact that the plasma waves with phase velocity $\sim c$ has energy and momentum densities $n_{o} m_{e} c^{2} \delta n_{e}^{2} / n_{o}^{2}$ and $n_{o} m_{e} c \delta n_{e}^{2} / n_{o}^{2}$.

$$
\delta \Psi_{1}=\sqrt{4 \pi m_{e} c^{2} n_{o} \frac{\omega_{p}}{\omega_{o}} \frac{\delta n_{e}}{n_{o}}}
$$

Using Equations (5) and (6) we get

$$
\frac{\partial \delta n_{e}}{\partial t}=\frac{-i \sqrt{2} n_{o} G \delta \Psi_{1}^{*} \delta \Psi_{o}}{4 \pi m_{e} c^{2} \hbar} \times \frac{\omega_{o}}{\omega_{p}}
$$


where * denotes complex conjugate. From Equations (5) and (6) we obtain the growth rate of stimulated scattering of neutrinos on plasma oscillations.

$$
\gamma=\sqrt{2} \frac{G}{\hbar} \sqrt{\frac{n_{o}\left|\delta \Psi_{o}\right|^{2} \omega_{o}}{4 \pi m_{e} c^{2} \omega_{p e}}}
$$

To determine the threshold of the instability we have to add the dissipation term to Equations (5) and (7).

These equations then become

$$
\begin{gathered}
\frac{\partial \delta \Psi_{1}}{\partial t}+\gamma_{\nu} \delta \Psi_{1}+\frac{1 \sqrt{2} G \delta n_{e}^{*} \delta \Psi_{o}}{\hbar}=0 \\
\frac{\partial \delta n_{e}}{\partial t}+\gamma_{p} \delta n_{e}+\frac{1 \sqrt{2} n_{o}^{2} G \delta \Psi_{1}^{*} \delta \Psi_{o}}{2 \pi \hbar m_{e} n_{o} c^{2}} \frac{\omega_{o}}{\omega_{p}}=0
\end{gathered}
$$

where $\gamma_{\nu}$ is the damping frequency for the neutrino waves $\left(=\frac{G^{2}(K T)^{5} c}{4 \pi^{2} \hbar^{7} c^{7}}\right)$ and $\gamma_{p}$ is the damping rate of the plasma oscillations.

The damping of the Langmuir plasmons $\gamma_{p}$ is the sum of collisional damping and Landau damping. For forward scattering, Landau damping is unimportant since the phase velocity of the plasma wave is close to $c$ which is necessary to maintain phase coherence with the neutrino field. Equations (10) and (11) give the solubility determinant as

$$
\left|\begin{array}{ll}
\gamma+\gamma_{\nu} & \frac{-1 \sqrt{2} G \delta \Psi_{o}}{\hbar} \\
\frac{-1 \sqrt{2} n_{o}^{2} G \delta \Psi_{o}}{4 \pi m_{e} n_{o} c^{2} \hbar \frac{\omega_{O}}{\omega_{p}}} & \gamma+\gamma_{p}
\end{array}\right|=0
$$

which yields the following growth rate with damping included

$$
\gamma=\frac{1}{2}\left\{-\left(\gamma_{\nu}+\gamma_{p}\right) \pm \sqrt{\left(\gamma_{\nu}-\gamma_{p}\right)^{2}+\alpha\left|\delta \Psi_{o}\right|^{2}}\right\}
$$

where $\alpha=n_{o} \omega_{o} / G^{2} / 2 \pi m_{e} c^{2} \omega_{p} \hbar$. Setting $\gamma=0$ gives the threshold for stimulated scattering of neutrinos off Langmuir plasmons

$$
\left|\delta \Psi_{o}\right|^{2}=\frac{4 \gamma_{p} \gamma_{\nu}}{\alpha}
$$

The true damping of the neutrino field is of course negligibly small if we only consider electron-neutrino scattering, resulting in an unrealistically low threshold and high growth rate. However, there is an effective damping rate due to dephasing of the neutrino field with the generated plasma wave. The neutrino field has a broad frequency spectrum since it is a thermal spectrum. Dephasing is very rapid except for forward scattering, where the scattered wave moves along with the incident wave (both at essentially the speed of light) and so phase coherence can be maintained for long times. In the forward direction there is 
still an important phase slippage due to the angular spread in the propagation direction for the neutrinos. This gives them a spread in velocities along the radial (forward) direction which is $c \Delta \theta^{2}$. We could modify our above treatment to include neutrino waves propagating with a spread in angles, however, a simpler way of dealing with the finite spectral width of the neutrino flux is to describe the neutrinos in terms of a wave kinetic equation for the neutrino number density given $\mathrm{by}^{7}$

$$
\frac{\partial N}{\partial t}+\frac{\partial \omega_{\nu}}{\partial \underline{k}} \cdot \underline{\nabla} N-\frac{\partial \omega_{\nu}}{\partial \underline{x}} \cdot \underline{\nabla}_{k} N=0
$$

where $N=\frac{|\Psi|^{2}}{4 \pi \omega_{\nu}}$ is the wave action or neutrino number density. The third term in Equation (14) represents the change in neutrino action or number density due to spatial changes in the neutrino frequency. This term represents a force acting on the neutrino's. In a plasma the frequency $\omega_{\nu}$ changes due to the presence of Langmuir plasmons and can be calculated from the expression of the refractive index in matter given by

$$
\varepsilon=1-\frac{\sqrt{2} G}{k \hbar c}\left\{n_{e}-\left(\frac{1}{2}-2 x_{\omega}\right) n_{e}+\left(\frac{1}{2}-2 x_{\omega}\right) n_{p}-\frac{1}{2} n_{n}\right\}
$$

where $x_{w} \equiv \sin ^{2} \theta_{w}=0.23$ and $\theta_{w}$ is the Weinberg angle, $n_{p}$ is proton density, and $n_{n}$ is neutron density, $n_{e}$ is electron density. For our case we need only keep the electron effect. In a plasma with Langmuir plasmons, the density $n_{e}$ is a function of position such that $n_{e}=n_{o}+\delta n_{o}(x)$ where $\delta n_{e}$ is the electron density perturbation due to Langmuir plasmons. Using Equation (15), we get

$$
\frac{\partial \omega_{\nu}}{\partial x}=\frac{\sqrt{2}}{2} \frac{\omega_{\nu}^{2}}{c k^{2}} \frac{G}{\hbar c} \frac{\partial \delta n_{e}}{\partial x}
$$

The coupling of the neutrino flux to Langmuir plasmons leads to a change in the neutrino number density $N=N_{o}+\delta N$. Linearizing Equation (14) by assuming $\delta N$ varies as $\exp i(\underline{q} \cdot \underline{x}-\Omega t)$ we get

$$
\delta N=\frac{i \frac{\sqrt{2}}{2} \frac{\omega_{k}^{2}}{c k^{2}} \frac{G}{\hbar} \frac{\partial \delta n_{e}}{\partial \underline{x}} \cdot \frac{\partial N_{e}}{\partial \underline{k}}}{\left(\Omega-\underline{q} \cdot \underline{v}_{g}\right)}
$$

where $v_{g}$ is the group velocity of the neutrino wave packet $(\approx c)$. Substituting Equation (17) into the equation for Langmuir waves leads to the following dispersion relation for cold Langmuir plasmons coupled to neutrinos

$$
\Omega^{2}-\omega_{p e}^{2}=-\frac{2 n_{o} \omega_{\nu} G^{2} \omega_{\nu}^{2}}{m_{e} c^{2} \hbar^{2} c^{2} k^{2}} \int \frac{q \underline{q} \cdot \frac{\partial N_{o}}{\partial \underline{k}}}{\left(\Omega-q \cdot v_{g}\right)} d \underline{k}
$$

(to include the effect of collisional Langmuir wave damping, one replaces $\Omega^{2}$ on the left-hand side or $\Omega\left(\Omega+i \gamma_{p}\right)$. 
The integral has a pole at q $\Omega=\underline{q} \underline{v}_{g}$ which gives rise to an imaginary component of $\Omega$ resulting in growth or damping, depending on sign of $\frac{\partial N_{0}}{\partial k}$ of Langmuir plasmons due to the intense neutrino flux.

The pole can also lie in a region where $\frac{\partial N_{0}}{\partial k}$ is zero. This is roughly the case for the situation of interest here where most of the spread in velocities parallel to $\underline{q}$ comes from the angular spread in direction of neutrino propagation. We can obtain an estimate of this growth rate by assuming the neutrinos are propagating at a small angle to $z$ with uniform angular distribution in this solid angle; we take $N\left(k_{\nu}\right)$ to be

$$
N\left(k_{\nu}\right)=\frac{\delta\left(\left|k_{\nu}\right|-k_{\nu o}\right) N_{o}}{2 \pi\left(1-\cos \theta_{o}\right)}
$$

where $\theta_{o}=a / R, a$ being the radius of the core and $R$ the distance from the center. We will assume that the most unstable mode has a phase velocity $\|$ to $z$ and just slower than $c \cos \theta_{o}$. Then the singularity in the denominator of Equation (18) lies in the region where $\frac{\partial N_{\varrho}}{\partial k}=0$.

Integrating Equation (18) by parts and using Equation (19), we get after integrating over $k$

$$
\Omega^{2}-\omega_{p e}^{2}=\frac{n_{o} \omega_{\nu}^{3} G^{2}}{m_{e} \hbar^{2} c^{3}} \frac{q^{2} N_{o}}{\left(1-\cos \theta_{o}\right)} \int \frac{\sin ^{3} \theta}{(\Omega-q c \cos \theta)^{2}} d \theta
$$

If we assume that the main contribution is close to $\theta_{o}$ because of $\sin ^{3} \theta$ dependance and replace $\cos \theta$ by $\cos \theta_{o}$ and take $\theta_{o}$ small so that $\theta \simeq \sin \theta$ this yields

$$
\Omega^{2}-\omega_{p e}^{2}=\frac{1}{2} \frac{n_{o} N_{o} G^{2} \omega_{\nu}^{3} q^{2} \theta_{o}^{2}}{m_{e} \hbar^{2} c^{3}\left(\Omega-q c+q \frac{c \theta_{o}^{2}}{2}\right)^{2}}
$$

Let $\Omega=\omega_{p}+\delta$, where $\delta$ is small, then $\Omega \simeq \pm \omega_{p}$ and $q c\left(1-\frac{\theta_{o}^{2}}{2}\right)=\omega_{p}$ gives us the following growth rate of Langmuir plasmons due to the intense neutrino flux

$$
I m \delta=\frac{\sqrt{3}}{2^{4 / 3}}\left[\frac{n_{o} N_{o} G^{2} \omega_{\nu}^{3} q^{2} \theta_{o}^{2}}{2 m_{e} c^{3} \hbar^{2} \omega_{p}}\right]^{1 / 2}
$$

For plasma densities found outside the core of $n_{o}=10^{30} \mathrm{~cm}^{-3}$ and neutrino intensities of $10^{34}(a / R)^{2} \mathrm{~W} / \mathrm{cm}^{2}$, we find the growth rate to be of the order of $10^{17} \mathrm{sec}^{-1}$ at distances of 10 core radii with the dependence on distance of $(a / R)^{4 / 3}$. From this calculation we see that the instability is quite strong and as a result we can expect the neutrino distribution to maintain a quasi-stable distribution for some distance out from the core. A quasilinear calculation of the neutrino motion out through the star is required, and we can expect a significant effect on supernova dynamics.

In this paper we show that it is possible to nonlinearly couple an intense neutrino flux to collective plasma oscillations. The nonlinear coupling results in absorption of the neutrino energy by Langmuir waves which heat the electrons through collisional damping thus 
increasing the electron pressure and helping to sustain the supernova shock. The energy distribution of the neutrinos should evolve as they propagate outwards. Plasmas can support many other types of waves besides Langmuir waves and many of these could also be involved in similar parametric instabilities. There is an extremely rich field of investigation here.

\section{Acknowledgments}

We would like to acknowledge fruitful discussions with H. Bethe and R.J.N. Phillips. This work was supported by NATO CGR 910316 and NSF PHY 91-21052. One of us (Su) would like to acknowledge the hospitality of Rutherford Appleton Laboratory where much of the early stages of this work was done. He also wants to acknowledge his visit to CERN where he had many interesting discussions.

\section{References}

1. Bethe, H.A., Rev. of Modern Physics, $\underline{62}, 801$ (1990).

2. Bethe, A.H. and J.R. Wilson, Astrophys. J., $\underline{295}, 14$ (1985).

3. Weinberg, S. Phys. Rev. Lett., 19, 1264 (1967).

4. Kaw, P.K., W.L. Kruer, C.S. Liu, and K. Nichikawa, Advances in Plasma Physics, Vol. 6, Part 1 (1986), John Wiley and Sons.

5. Bethe, H.A., Phys. Rev. Lett., $\underline{56}, 1305$ (1986).

6. Bowler, M.G., Femtophysics, A Short Course on Particle Physics, Pergamon Press, (1990).

7. Tappert, F.D., Siam Rev. (Soc. Ind. Appl. Math), $\underline{13}, 281$ (1971). 


